\title{
ARTICLE
}

\section{Long-term paired associative stimulation can restore voluntary control over paralyzed muscles in incomplete chronic spinal cord injury patients}

\author{
Anastasia Shulga ${ }^{1,2}$, Pantelis Lioumis ${ }^{2}$, Aleksandra Zubareva ${ }^{2}$, Nina Brandstack ${ }^{3}$, Linda Kuusela ${ }^{3,4}$, Erika Kirveskari ${ }^{5}$, \\ Sarianna Savolainen ${ }^{6}$, Aarne Ylinen ${ }^{1,7}$ and Jyrki P Mäkelä ${ }^{2,7}$
}

Emerging therapeutic strategies for spinal cord injury aim at sparing or restoring at least part of the corticospinal tract at the acute stage. Hence, approaches that strengthen the weak connections that are spared or restored are crucial. Transient plastic changes in the human corticospinal tract can be induced through paired associative stimulation, a noninvasive technique in which transcranial magnetic brain stimulation is synchronized with electrical peripheral nerve stimulation. A single paired associative stimulation session can induce transient plasticity in spinal cord injury patients. It is not known whether paired associative stimulation can strengthen neuronal connections persistently and have therapeutic effects that are clinically relevant. We recruited two patients with motor-incomplete chronic (one para- and one tetraplegic) spinal cord injuries. The patients received paired associative stimulation for 20-24 weeks. The paraplegic patient, previously paralyzed below the knee level, regained plantarflexion and dorsiflexion of the ankles of both legs. The tetraplegic patient regained grasping ability. The newly acquired voluntary movements could be performed by the patients in the absence of stimulation and for at least 1 month after the last stimulation session. In this unblinded proof-of-principle demonstration in two subjects, long-term paired associative stimulation induced persistent and clinically relevant strengthening of neural connections and restored voluntary movement in previously paralyzed muscles. Further study is needed to confirm whether long-term paired associative stimulation can be used in rehabilitation after spinal cord injury by itself and, possibly, in combination with other therapeutic strategies.

Spinal Cord Series and Cases (2016) 2, 16016; doi:10.1038/scsandc.2016.16; published online 14 July 2016

\section{INTRODUCTION}

There is a need for safe, noninvasive treatments to be applied at the acute and chronic stages after spinal cord injury (SCI). Therapeutic strategies being developed for the acute stage of $\mathrm{SCl}$ aim at sparing or restoring at least part of the corticospinal tract. ${ }^{1}$ In parallel, it is crucial to develop approaches that would strengthen the weak connections that are spared or restored. In cell and animal models, plastic changes that lead to strengthening of neuronal connectivity (long-term potentiation (LTP)) can be induced by electrical stimulation. ${ }^{2}$ In humans, LTP-like plasticity can be achieved by noninvasive brain stimulation.

One of the noninvasive brain stimulation methods extensively studied in healthy subjects is paired associative stimulation

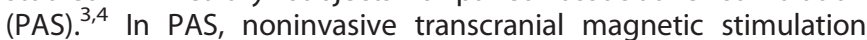
(TMS) is synchronized with noninvasive peripheral nerve electrical stimulation (PNS); signals are timed to coincide at synapses at cortical $^{3}$ or the spinal cord $^{5-7}$ level to enhance corticospinal neuron excitability. A single PAS session applied to one nerve can induce transient (up to $90 \mathrm{~min})^{4}$ plasticity in healthy subjects; importantly, one work in healthy subjects has shown that the effect of the protocol repeated for 3 days lasts for at least 2 days after the last stimulation session. ${ }^{8}$ Transient plasticity has also been induced by PAS in $\mathrm{SCl}$ patients, ${ }^{9,10}$ but effects of repetitive PAS in $\mathrm{SCl}$ patients have never been reported.

PAS combines beneficial effects of both TMS and PNS while avoiding several detrimental effects. For example, PAS pulse pairs are delivered once every $5 \mathrm{~s}$. Repetitive TMS (rTMS) without concomitant PNS can have positive effects in incomplete $\mathrm{SCl}$ patients for 2-3 weeks; ${ }^{11,12}$ however, in SCl patients, high-intensity TMS is needed to effectively activate the corticospinal tract, ${ }^{13}$ and long-term use of high-frequency and high-intensity rTMS may be contraindicated for SCI patients with head injury, as rTMS reduces the seizure threshold. ${ }^{13,14}$ In contrast, PAS utilizes less risky low-frequency TMS.

PNS without TMS (for example, peripheral nerve somatosensory stimulation or vibration) is also beneficial in the rehabilitation of incomplete $\mathrm{SCl}$ patients. ${ }^{15}$ They are applied only for muscles that have remaining voluntary activity and must be applied simultaneously with voluntary activations of targeted muscles. ${ }^{15-18}$ Studies of rTMS and transcranial direct cortical stimulation are also based on patients with preserved voluntary activity in the muscles under investigation. ${ }^{11,12,18}$ PAS induces plasticity in humans without concomitant effort by the subject; ${ }^{4}$ we thus hypothesized that long-term PAS could be a

\footnotetext{
${ }^{1}$ Clinical Neurosciences, Neurology, University of Helsinki and Helsinki University Hospital, Helsinki, Finland; ${ }^{2}$ BioMag Laboratory, HUS Medical Imaging Center, University of Helsinki and Helsinki University Hospital, Helsinki, Finland; ${ }^{3}$ Department of Radiology, HUS Medical Imaging Center, University of Helsinki and Helsinki University Hospital,

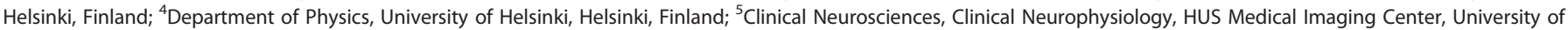
Helsinki and Helsinki University Hospital, Helsinki, Finland and ${ }^{6}$ Validia Rehabilitation Center, Helsinki, Finland.

Correspondence: A Shulga (anastasia.shulga@helsinki.fi)

${ }^{7}$ These authors contributed equally to this work.

Received 4 February 2016; revised 20 May 2016; accepted 28 May 2016
} 
technique that could affect also those muscles which are fully paralyzed.

Ability of PAS to strengthen neuronal connections persistently and induce clinically relevant effects after $\mathrm{SCl}$ is not known. To address this question, we recruited two chronic SCl patients with incomplete motor affision. We show that spinal PAS consisting of TMS combined with peripheral trains of high-frequency stimulation, applied for a sufficient period of time, is capable of restoring voluntary control over previously paralyzed muscles.

\section{PATIENTS AND METHODS}

Patients

The study was approved by the Ethics Committee of Helsinki University Hospital. Both patients signed an informed consent before the intervention. Both were healthy before the accident that led to the SCI. Both had standard decompression/fixation surgery immediately after the accident. Methylprednisolone was not given (according to national guidelines). The standard-of-care rehabilitation was initiated as soon as possible and continued before the study and in parallel with the study. The amount of physical rehabilitation was not increased during the study. After the study, the newly acquired movements were incorporated into the rehabilitation by the patients' own physiotherapists. The magnetic resonance imaging (MRI) images of the patients' spinal cord at the acute stage and at the beginning of the project are presented in Figure 1.

A 31-year-old paraplegic female, (patient A; American Spinal Injury Association Impairment Scale (AIS) C, height $163 \mathrm{~cm}$, right-handed; neurological level L1) sustained $\mathrm{SCl}$, due to a falling accident (L1 burst fracture) 2 years and 2 months before enrollment in the study, resulting in incomplete paraplegia. Figures $1 \mathrm{a}$ and $\mathrm{b}$ demonstrate that the area of the acute damage and the chronic posttraumatic atrophy is above the end of conus medullaris; the patient thus sustained $\mathrm{SCl}$ and not a cauda equina injury. Immediately after injury, she had no motor activity in the legs, and the sensory level was at L1 bilaterally. During the first 6 months after injury, she regained activity in the lower limb muscles above the knee level, but remained fully paralyzed below the knee level; there was no further neurological improvement for at least 1 year before the beginning of the study. The lower-extremity motor score before intervention was right/left: L2 4/3, L3 5/4 and L4-S1 0/0. Her standard-of-care rehabilitation program consists of physiotherapy two times per week. Patients with SCl at the thoracolumbar level often have preserved volitional movements in the proximal muscles and absent responses in the distal muscles $;{ }^{14}$ our patient thus is a typical example of this group. The spinal cord independence measure (SCIM) score of the patient is 76; she uses a wheelchair.

A 53-year-old male (patient B; AIS C, height $174 \mathrm{~cm}$, right-handed; neurological level $\mathrm{C} 3$ ) sustained $\mathrm{SCl}$ due to a traffic accident (C3-5 fractures) 2 years and 3 months before the study, resulting in incomplete tetraplegia. He had some preserved muscle activity in all muscle groups. However, part of his hand muscles were fully paralyzed, and he was not able to grasp (see Supplementary Table for detailed motor scores of the upper limbs). The AIS motor score of the upper limbs was stable during the year before the study; the upper-extremity right/left motor score was: C5 1/1, C6 1/1, C7 1/2, C8 $1 / 3$ and T1 1/1. A small syrinx cavity was visualized in the MRI of the spinal cord (Figure 1d). The patient was clinically diagnosed with a mild brain injury, but the head MRI was normal. His standard-of-care rehabilitation consists of physiotherapy three times per week and occupational therapy two times per week (given on the same days as physiotherapy), as well as $20-30$ min neuromuscular electrical stimulation three times per week to biceps, shoulders, buttocks, wrists and abdomen. The neuromuscular electrical stimulation is given symmetrically and equally to both sides. The symptoms of autonomic dysreflexia were extremely rare. The SCIM score is 0 ; he uses a wheelchair and needs an assistant.

\section{Medication and nutrition of the patients}

To ensure that lack of vitamins or minerals would not constrain a possible therapeutic effect, both patients were instructed to take daily a multivitamin tablet at a standard dose. At the beginning of the intervention, patient A used nortriptylline $10 \mathrm{mg}$ and pregabalin $550 \mathrm{mg}$ per day. During the first week of the intervention, she could reduce the pregabalin dose to $300 \mathrm{mg}$ per day and, starting from week 6, to $225 \mathrm{mg}$ per day. Patient $B$ used mirtazapin $30 \mathrm{mg}$, temazepam $10 \mathrm{mg}$, clonazepam a

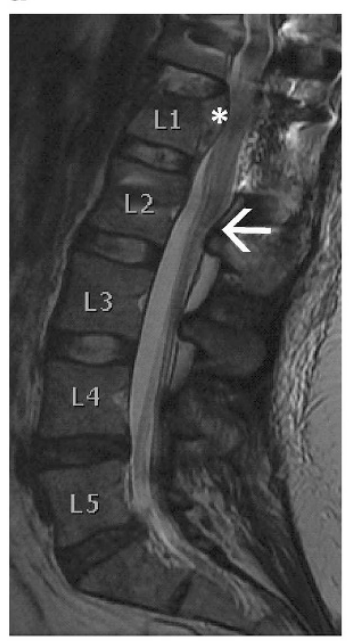

C

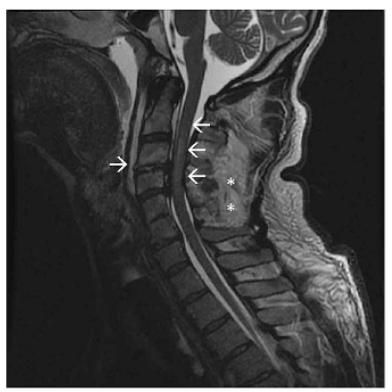

b

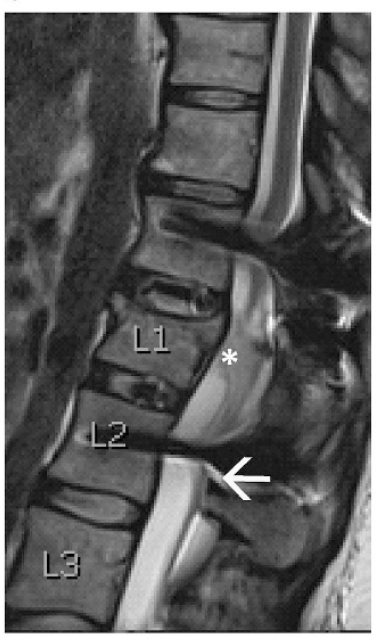

d

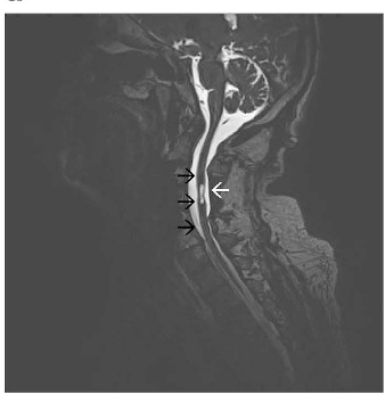

Figure 1. Spinal MRI from patients $A$ and $B$ at the acute $(\mathbf{a}, \mathbf{c})$ and chronic (b, d) stages. (a) Patient A. Sagittal T2-weighted fast spin-echo (FSE) image of the lumbar spine achieved with 1.5-T MRI scanner. White star: L1 burst fracture and abnormal narrowing of the spinal canal due to fracture displacement. White arrow: conus medullaris ends at the level of $L 2$ vertebra. The fractured $L 1$ was stabilized with transpedicular fixation through Th12 and L2. The image was taken 1 week after the injury (unrelated to the research project). (b) Patient A. Sagittal T2-weighted FSE image of the lumbar spine, 3-T MRI scanner. Posttraumatic atrophy of the distal spinal cord is visualized (white star). White arrow: the end of conus medullaris (indicated with white arrow in (a)). The scan was taken before the first stimulation of the right leg. (c) Patient B. Sagittal T2-weighted FSE image of the cervical spine achieved with 1.5-T MRI scanner. There is T2 signal increase representing spinal cord contusion at the C2-C4 level (leftpointing white arrows). C4 and C5 processus spinosi are fractured (white stars). Soft-tissue edema as well as disruption of the C3/4 anterior longitudinal ligament and discus (right-pointing white arrow) are visualized. The image was taken 1 day after the injury (unrelated to the research project). (d) Patient B. Sagittal T2-weighted threedimensional image of the cervical spine, 3-T MRI scanner. Posttraumatic atrophy of the spinal cord (black arrows) and trauma-induced syringomyelia (white arrow) at the C3/4 level are visualized. The scan was taken before the first stimulation.

$2 \mathrm{mg}$ and baclofen $60 \mathrm{mg}$ daily (only drugs affecting the central nervous system are listed).

Timetable of the stimulation

The timetable is described in Figure 2. The 2- and 1-weeks gaps were introduced into the program of patient $A$, because she was traveling.

\section{Transcranial magnetic stimulation}

TMS was delivered by an eXimia magnetic stimulator (Nexstim Ltd., Helsinki, Finland). The stimulation frequency was $0.2 \mathrm{~Hz}$ (Figure 3a). The stimulation intensity was $90 \%$ of the stimulator output for patient $A$ and $100 \%$ stimulator output for patient B. Our MRI-guided TMS navigation 


\begin{tabular}{|c|c|}
\hline \multicolumn{2}{|c|}{$\begin{array}{l}\text { Patient A } \\
\text { stimulation }\end{array}$} \\
\hline & $\begin{array}{l}2 \text { years } 2 \text { months: } \\
\text { standard-of-care rehabilitation }\end{array}$ \\
\hline $1-2$ & 2 weeks: PAS 5 times a week, left leg \\
\hline $3-9$ & 7 weeks: PAS 3 times a week ${ }^{*}$, left leg \\
\hline $10-11$ & $\begin{array}{c}2 \text { weeks: PAS } 5 \text { times a week, right leg } \\
\text { and } 3 \text { times a week, left leg }\end{array}$ \\
\hline $12-13$ & 2 weeks: PAS 3 times a week ${ }^{*}$, both legs \\
\hline \multirow{3}{*}{14} & 2 weeks: no stimulation \\
\hline & 1 week: PAS 2 times a week, both legs \\
\hline & 1 week: no stimulation \\
\hline $15-21$ & 7 weeks: PAS 3 times a week, both legs \\
\hline
\end{tabular}

stimulation

Patient B

\begin{tabular}{l|l|} 
week & $\begin{array}{l}2 \text { years } 3 \text { months: } \\
\text { standard-of-care rehabilitation }\end{array}$ \\
\cline { 2 - 2 } & $\begin{array}{r}\text { 2 weeks: PAS } 5 \text { times a week, right hand } \\
\text { and control PNS, left hand }\end{array}$ \\
\hline 3-1 & $\begin{array}{r}10 \text { weeks: PAS } 3 \text { times a week, right hand } \\
\text { and control PNS, left hand }\end{array}$ \\
\hline $13-14$ & 2 weeks: PAS 5 times a week, left hand \\
\hline 15-24 & 10 weeks: PAS 3 times a week, left hand \\
\hline
\end{tabular}

Figure 2. Time course of the experiment. *During weeks 7 and 13 , PAS was given only two times per week, **during weeks 5 and 12 , stimulation was given two times per week.

a
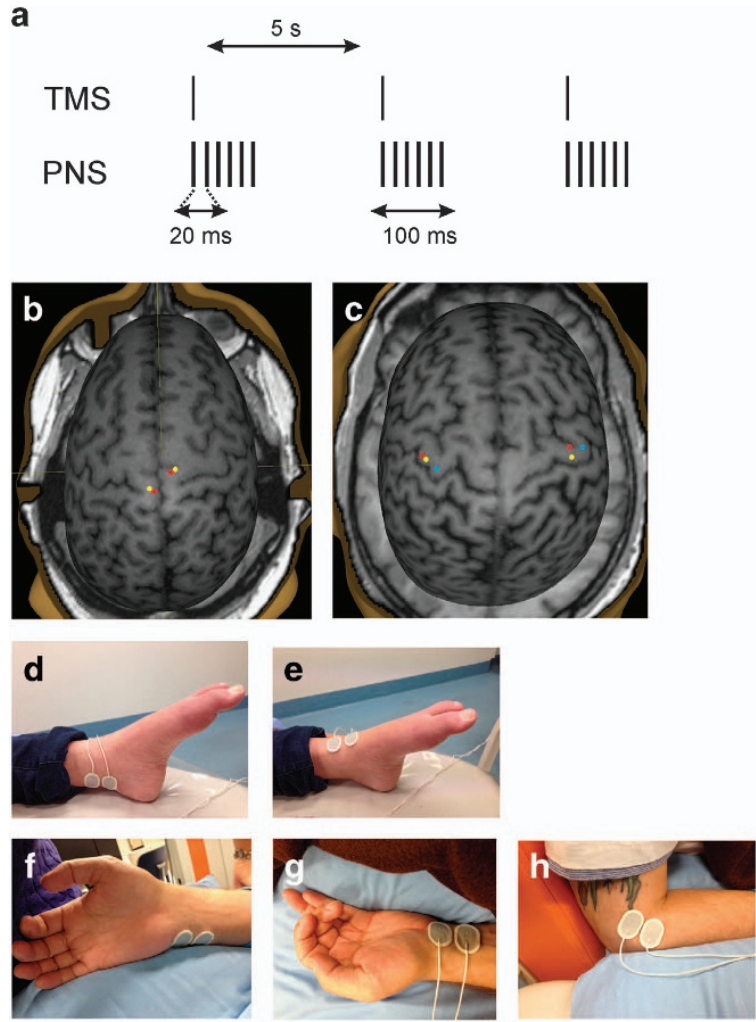

Figure 3. PAS protocol and the sites of stimulation. (a) Schematic representation of stimulation protocol. PNS pulse width: $1 \mathrm{~ms}$. (b) Cortical sites of TMS for patient A were paired with stimulation of the contralateral: yellow-peroneal nerve, red-tibial nerve. (c) Cortical sites of TMS for patient B were paired with stimulation of the contralateral: red-median nerve, yellow-ulnar nerve and blue-radial nerve. (d-h) Peripheral electrode location for stimulation of the: (d) tibial nerve (medial side of the ankle behind the medial malleolus) (e) peroneal nerve (frontal side of the ankle), (f) ulnar nerve (medial side of the wrist), (g) median nerve (middle of the wrist) and (h) radial nerve (lateral side of the arm). system (Navigated Brain Stimulation 4.3 (NBS 4.3), Nexstim Ltd., Helsinki, Finland) ensured that the TMS stimulation targets of the PAS protocol were the very same at every stimulation session. The sites of cortical stimulation were selected separately for each peripheral nerve to be paired in PAS.

For patient $\mathrm{B}$, the targets were selected by mapping the motor cortex. We selected the sites where the motor-evoked potentials (MEPs) were most readily elicited from the abductor pollicis brevis, abductor digiti minimi and brachioradialis muscles to pair them with the median, ulnar and radial nerve stimulations, respectively. For patient $A$, such mapping was challenging, because the representation area of the quadriceps muscle had spread over almost the entire lower limb representation area in the primary motor cortex. Consequently, stimulation of several sites caused quadriceps contraction and movement artifacts in the MEPs. We selected the sites separately for the peroneal and tibial nerves, partly by predicting the location of the optimal sites, based on brain anatomy, and partly by examining the MEPs recorded from the abductor hallucis muscle for the tibial nerve, and the extensor digitorum brevis/tibialis anterior (TA) muscles for the peroneal nerves. From these MEPs, we selected as targets the points that repeatedly produced similar responses.

\section{Electrical peripheral nerve stimulation}

Dantec Keypoint electroneuromyography device (Natus Medical Inc. Pleasanton, CA, USA) and surface electrodes (Figures 3d-h) (Neuroline 720; Ambu A/S, Ballerup, Denmark) were used for PNS delivered as $50-\mathrm{Hz}$ trains of 1-ms biphasic square-wave pulses for $100 \mathrm{~ms}$ (Figure 3a). The stimulation intensity was $25 \mathrm{~mA}$. During radial nerve stimulation, the electrodes were gently pressed against the skin.

\section{Paired associative stimulation}

We aimed at the simultaneous arrival of the pre- and postsynaptic volleys at the corticomotoneuronal synapses. In patient $B$, we measured the MEP latencies and F-responses from the abductor pollicis brevis muscle to estimate the interstimulus interval (ISI) for PAS involving the median nerve, from the abductor digiti minimi muscle for PAS involving the ulnar nerve, and from the brachioradialis muscle for PAS involving the radial nerve. We then calculated the ISIs between the TMS and the first pulse of the PNS train using the formula (F-latency minus MEP latency), as described in our previous work. ${ }^{19}$ According to these calculations, for the right hand we used the -1ms interval (TMS before PNS) for PAS involving the ulnar and radial nerves and the +1-ms (TMS after PNS) for PAS involving the median nerve. For the left hand, we used the ISIs of -4 (ulnar and median) and -5 (radial) ms.

In patient $\mathrm{A}$, we did not calculate the ISI, because the determination of her MEP latency was challenging (see above). We chose a 0-ms ISI (see 
a
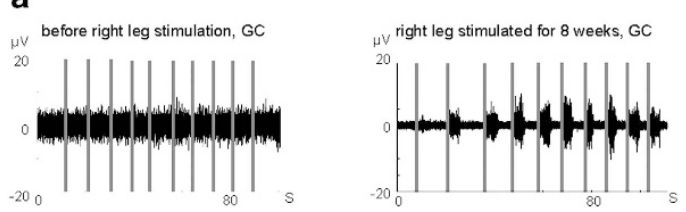

right leg stimulated for 12 weeks, GC
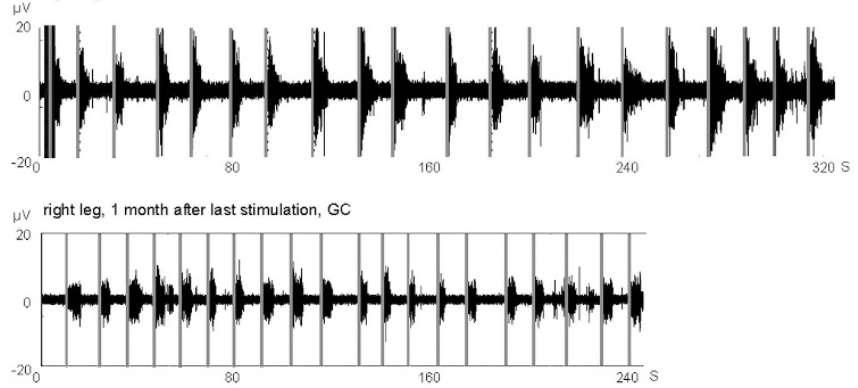

C
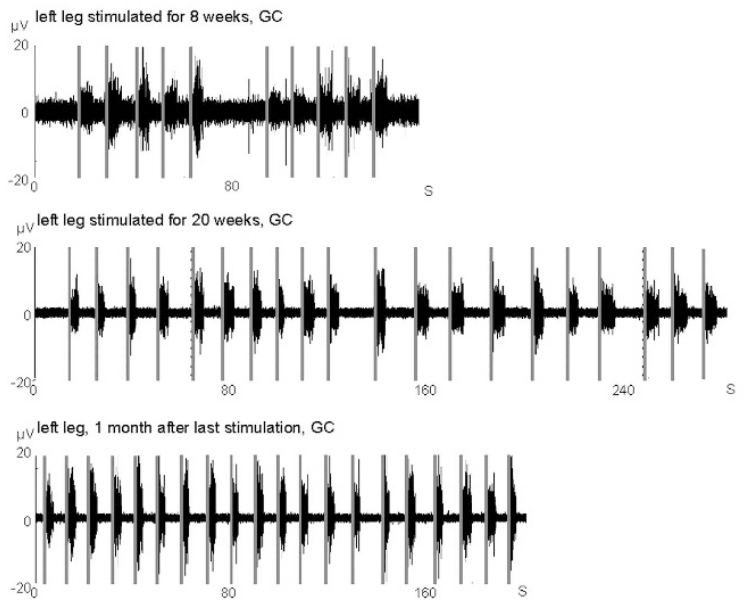

b

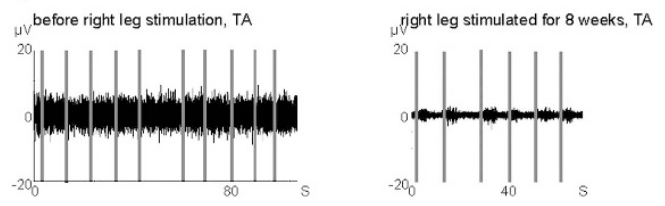

right leg stimulated for 12 weeks, TA

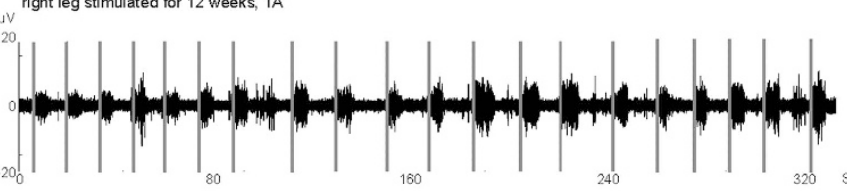

right leg, 1 month after last stimulation, TA

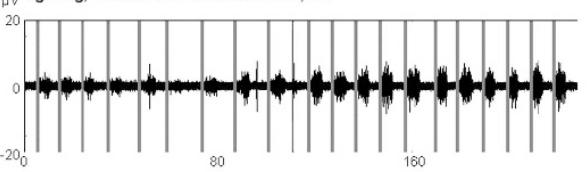

d
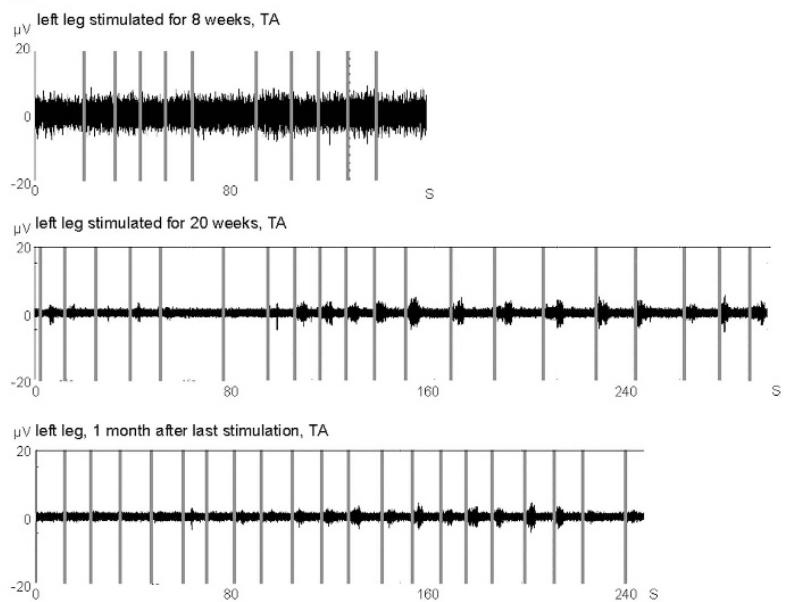

Figure 4. EMG recordings from patient A. Vertical gray lines: event of the command to move the foot. (a) Right medial gastrocnemius (GC) muscle during plantarflexion (foot down). (b) Right TA muscle during dorsiflexion (foot up). (c) Left medial GC during plantarflexion. (d) Left TA during dorsiflexion. See also Supplementary Videos 1 and 2. See Figure 5 for control recordings.

Discussion section for the choice of protocol including $50-\mathrm{Hz}$ PNS and its possible effect on ISIs). The PNS and TMS were triggered by Presentation software (Neurobehavioral Systems Inc., Albany, CA, USA).

Both patients received PAS for $1 \mathrm{~h}$ per limb. In patient $A$, the duration was $30 \mathrm{~min}$ per nerve, and the stimulation was applied to the tibial and peroneal nerves. In patient $B$, the stimulation was applied for 20 min to each of the median, radial and ulnar nerves.

During PAS and during control PNS (see below), the patients were instructed to focus on the movements (to have the movement on their mind; not to attempt the performance of the movement) produced by the muscles innervated by the stimulated nerves.

Selection of side of the treatment

For patient $A$, the order of the treatment (first left leg, then right leg) was selected randomly. For patient $\mathrm{B}$, we selected the right dominant hand for PAS and left hand for control PNS (see below), since the patient was presumed to be more motivated for a long pilot treatment if possible positive outcome would occur in the dominant hand.

\section{Control PNS}

To ensure that the observed effects were not due to PNS only, we applied PAS first only to the right hand of patient B. For the left hand, we applied only PNS of the median nerve with the same settings as in PAS, but without concomitant TMS, for 20 min immediately after the PAS sessions of all three nerves for the right hand. After 12 weeks of control PNS stimulation, we continued with 12 weeks of PAS for the left hand.
Since we did not include the corresponding control condition for patient A, we performed 3 PAS and 3 sessions with PNS only on age- and gendermatched healthy control individual (32-year-old female). PAS protocol was the same as in patients and was applied for $20 \mathrm{~min}$ to right tibial nerve/left motor cortex. TMS intensity was $100 \%$ stimulator output and PNS intensity $15 \mathrm{~mA}$. MEPs were measured from right abductor hallucis muscle immediately after and $1 \mathrm{~h}$ after PAS or PNS. MEP amplitudes did not increase immediately after PAS $(112 \pm 12 \%, n=3$ sessions), but did increase significantly $1 \mathrm{~h}$ after PAS ( $130 \pm 1 \%, P=0.01$ by paired sample $t$-test, $n=3$ sessions). After PNS alone there was no increase in MEP amplitudes immediately after $(90 \pm 2 \%, n=3$ sessions) and $1 \mathrm{~h}$ after $(72 \pm 15 \%, n=3$ sessions) PNS.

\section{Clinical evaluation of the patients}

A physician evaluated the sensory scores. Two physiotherapists, with long experience in working with $\mathrm{SCl}$ patients, evaluated the motor scores of each muscle, manually immobilizing other muscles during testing. The same physiotherapist and the same physician evaluated all motor scores of one patient before, during and after intervention. Standard scales of the AIS examination sheet were used. The evaluators were not blinded to the treatment, but did not have the results of the previous evaluations available during examination.

Please see Supplementary Videos.

\section{EMG and MEP recordings}

An electromyogram (EMG) was recorded with the built-in EMG device of our TMS system (eXimia NBS 4.3). The examiner requested the patient to 
move, and simultaneously pressed the foot switch to mark the onset of the movement attempt in the EMG recording. The EMG was processed offline, using Python (Python Software Foundation, Beaverton, OR, USA). The signal was band-pass-filtered between 20 and $300 \mathrm{~Hz}$ with a Chebyshev type II filter and notch-filtered at $50 \pm 1 \mathrm{~Hz}$. The MEPs were recorded with the same equipment; 30 MEPs were sampled at an interval of $3.3 \mathrm{~s}$ and quantified offline with Nexstim NBS 4.3 software. In calculating the average amplitude values, the stimuli eliciting no MEPs were counted as 0 . For graphical presentation of the results, the MEP traces were averaged using MATLAB (MathWorks Ltd., Nattick, MA, USA) software. All measurements were done at least 2 days after PAS session.

\section{Magnetic resonance imaging}

Project-related MRI images at the chronic stage were acquired with a 3-T Siemens Verio (Siemens Healthcare, Erlangen, Germany) scanner. The imaging protocol included structural sequences of the head and spinal cord at the C2-C3 level and the area of injury.

\section{Statement of ethics}

We certify that all applicable institutional and governmental regulations concerning the ethical use of human volunteers were followed during the course of this research.

\section{RESULTS}

Motor function

For Patient A, we applied PAS that included the tibial and peroneal nerves (Figures $3 d$ and e), first to the left leg and later to both legs regularly three times per week (Figure 2 ). The cortical stimulation site paired with each nerve was selected separately (Figure $3 b$ ).
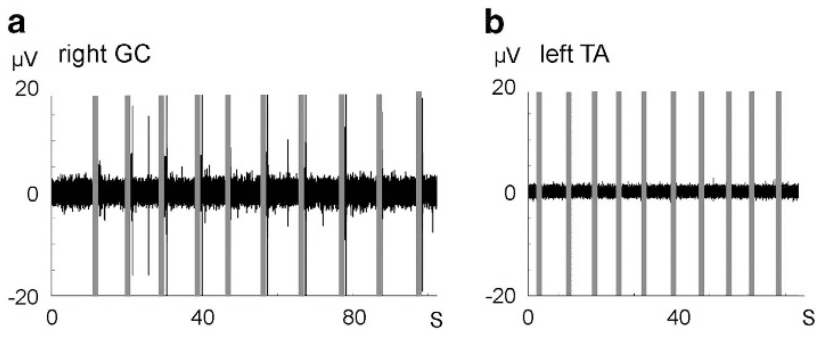

Figure 5. Control recordings of the EMG traces shown in Figure 4. (a) EMG from right medial gastrocnemius (GC) during medial inversion of the hip. The recording was made at the same session as the one shown in Figure 4a (12-week trace). (b) EMG from left TA during contraction of quadriceps femoris muscle without attempt to dorsiflex the ankle. The recording was made in the same session as the one shown in Figure $4 d$ (20-week trace).
After the injury, the patient had been able to imagine moving the left ankle, but lost this ability some months after the injury. Four weeks after the first PAS of the left leg, the patient reported that she could again imagine the movements. At 5-6 weeks we observed first movements at foot dorsiflexion and plantarflexion. At 8 weeks after the first PAS, we recorded EMG activation during voluntary plantarflexion (Figure 4c). The EMG activation during dorsiflexion could be observed at week 20 (Figure 4d).

During the left leg PAS of patient $A$, the right leg remained paralyzed below the knee level. The ability to move the right ankle emerged 3-4 weeks after PAS onset for the right leg, and we recorded EMG activation at 8 weeks (Figures $4 a$ and b). Importantly, the patient was able to move the feet also in the absence of stimulation (Supplementary Videos 1 and 2). At the 1-month evaluation after the last PAS session, the ability to move was preserved (Figure 4). The control EMGs are shown in Figure 5. The motor scores of the lower limb muscles not innervated by the peroneal and tibial nerves did not improve.

For patient B, three different left hemisphere cortical stimulation sites (Figure $3 \mathrm{c}$ ) were paired with the stimulation of the right median, ulnar and radial nerves. We also stimulated the median nerve of the left hand without concomitant TMS to investigate the impact of PNS alone. We observed a rapid increase in the motor score of the right hand muscles (Figure 6 and Supplementary Table). The patient regained the ability to grasp objects (Supplementary Video 3). PNS alone did not affect the muscles innervated by the left median nerve (Figure 6 and Supplementary Table). At the evaluation 1 month after the last PAS session, the increase in the motor score was preserved (Figure 6 and Supplementary Table). After the 12-week control PNS of the left hand was completed, we gave PAS to the same hand for 12 weeks. We observed an increase in the motor score of the left hand (Figure 6 and Supplementary Table). After 12 weeks of PAS, there was also a robust improvement in the muscles that were not innervated by the radial, median or ulnar nerves (Supplementary Table).

\section{MEP measurements}

In patient $A$, the MEP amplitudes in the TA muscle were decreased to $55 \pm 20 \%$ immediately $(0 \mathrm{~min})$ after PAS, whereas in the gastrocnemius muscle they remained at $97 \pm 8 \%$. In patient $B$, the MEP amplitude in the abductor pollicis brevis muscle decreased to $32 \pm 6 \%$ (for each muscle, $n=3$ measurements on different days, 30 MEPs per measurement). The long-term outcome of the PAS, however, was a robust growth in the MEP amplitudes over time (Figure 7). This increase in MEP amplitudes

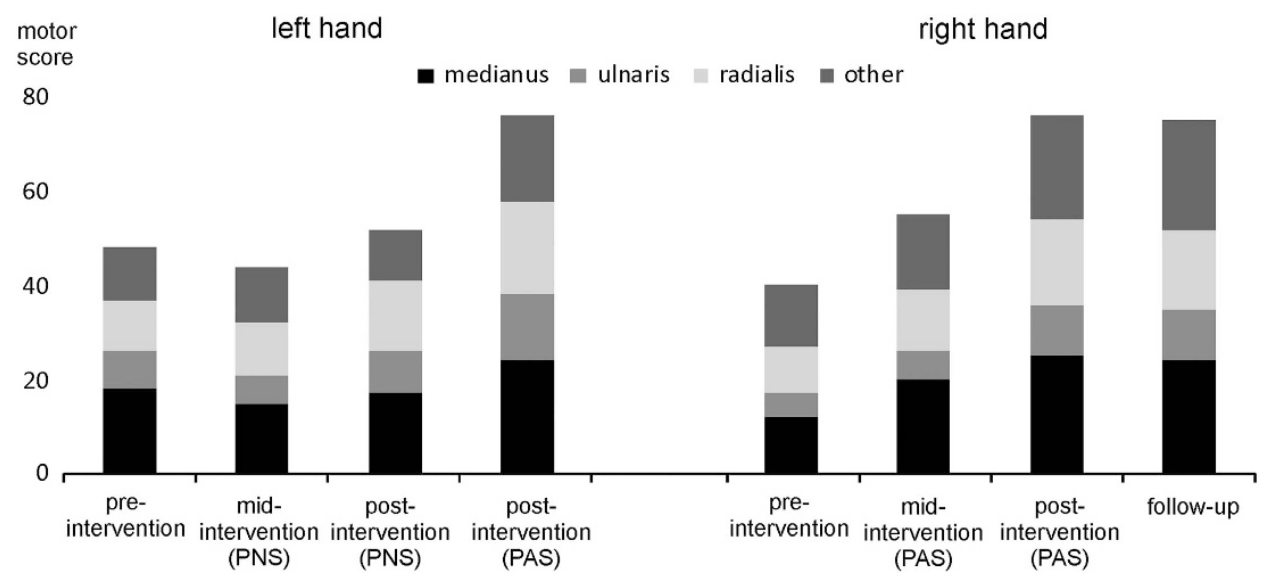

Figure 6. Motor score of the right and left upper limbs of patient B before (pre-intervention) and 7 (mid-intervention) and 12 (post-intervention) weeks after the first stimulation. Follow-up: 1 month after the last stimulation session. Scores for median, ulnar, radial and other nerves are shown separately. See Supplementary Table for details. See also Supplementary Video 3. 
a

GC Week 5

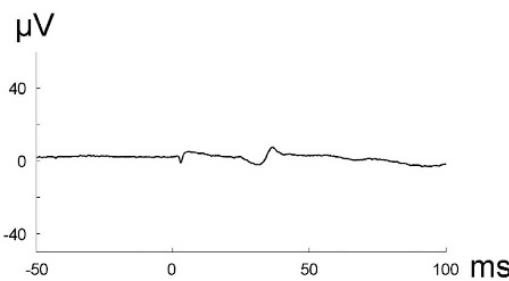

Week 21

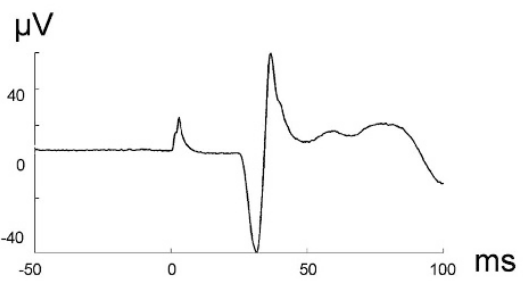

TA

Week 5

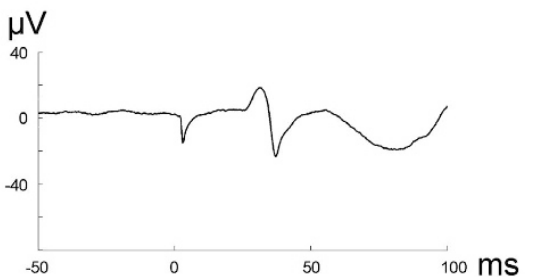

Week 20

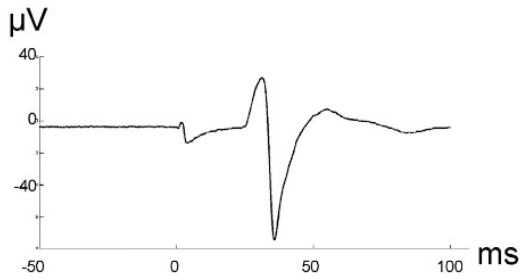

BR

Pre-intervention

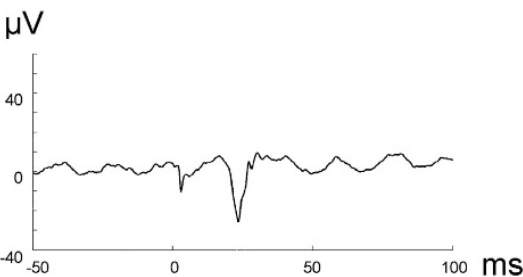

Week 12

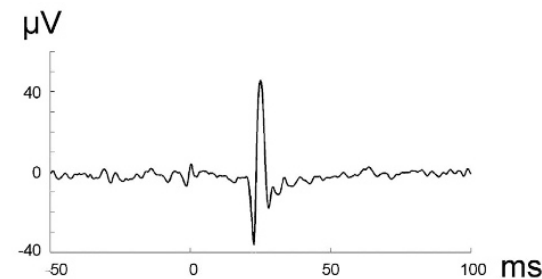

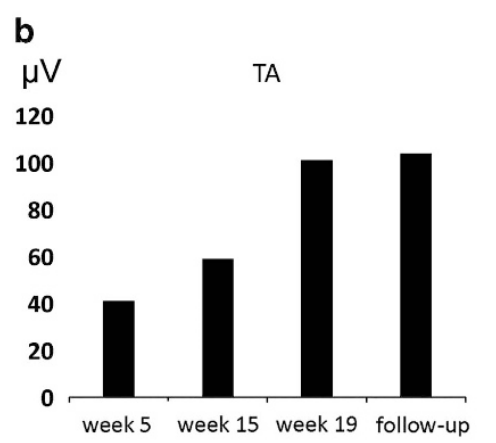

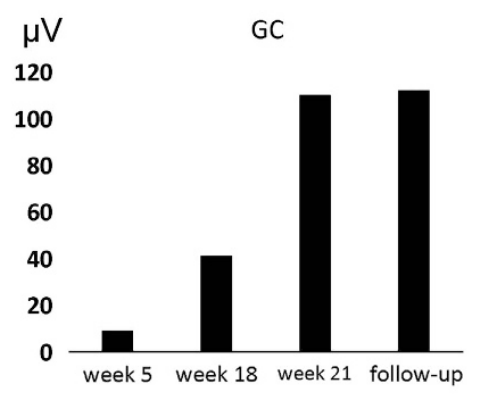

c
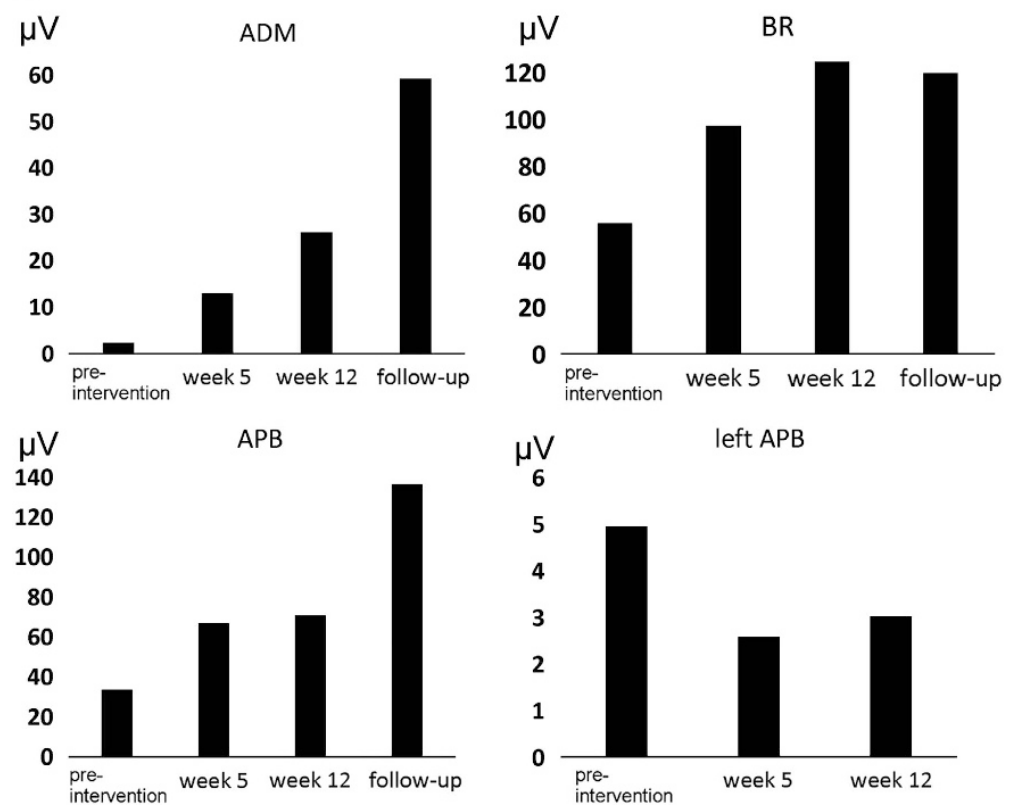

Figure 7. Neurophysiological measurements from both patients. (a) MEP recordings from patient A (left leg) and patient B (right arm). Each trace is an average of 30 MEPs taken in a row at a 3.3-s ISI. BR, brachioradialis muscle (patient B); GC, gastrocnemius muscles; TA, TA muscle (patient A). Short latencies of MEPs from patient A are explained by the short height of the patient $(163 \mathrm{~cm})$; the latency for the vastus medialis muscle in the left leg was $24 \mathrm{~ms}$. (b, c) MEP amplitudes during different weeks of stimulation. Follow-up: 1 month after the last stimulation session. The values are averages of 30 MEPs taken in a row. If no MEP was evoked by a stimulus, the amplitude was counted as 0. The values thus represent both amplitude and persistence of the MEPs. (b) MEPs in the paraplegic individual (left leg). (c) MEPs in the tetraplegic individual (right arm), ADM, abductor digiti minimi. Left APB: recordings during control PNS. 
persisted for at least 1 month after the last stimulation session (Figures $7 \mathrm{~b}$ and $\mathrm{c}$ ). The MEP latencies decreased by 1-1.6 ms in patient $B$ (but not after the control PNS), and remained the same in patient $A$.

\section{Neuropathic pain}

Before PAS, patient A reported almost daily throbbing bilateral pain in close proximity to the L2 sensory level. The level of pain was 3-6 on the Visual Analog Scale (VAS; 0-no pain; 10-worst possible pain). During the first week after PAS, the pain diminished bilaterally, although only the left leg/right cerebral hemisphere was stimulated. During the 6-month experiment, the patient experienced pain (VAS 4-5) only on 11 days (half of these 11 days were associated with an infection). During the 2 weeks of no stimulation (Figure 2), the pain did not reappear. During the follow-up period (1 month), the patient experienced pain (VAS 5) during 4 days, the pain being associated only with provoking stimuli.

Before the experiment, patient B reported pain of VAS 7 in the right shoulder and scapular area about three times per week. After 10 PAS sessions, the pain occurred about once in 2 weeks (VAS 7), and only in the shoulder area. During the follow-up period (1 month), the incidence and area of pain did not increase.

\section{Sensory scores}

The treatment did not affect the sensory scores significantly. In Patient A, the C2-L1 scores were normal (grade 2 for each segment in light-touch and pin-prick scores). In the L2-S5 area, the sum of light-touch and pin-prick scores was 7 before intervention, 8 at week 8 and 9 at week 20. The patient did regain the ability to feel the ankle movements. In patient $B$, the sum of light-touch and pin-prick scores in the C2-T10 area on the right/left sides was $21 / 16$ before and 20/14 after the intervention.

\section{Autonomic functions and spasticity}

The patients reported no effects on autonomic functions. Spasticity, assessed by the modified Ashworth scale, was not significantly reduced. Patient A had no spasticity before or after the intervention. The level of spasticity in patient $B$ varied daily.

\section{Adverse effects}

Both patients reported occasional transient musculoskeletal pain in the back or neck region, resulting from $1.5-3 \mathrm{~h}$ of sitting in the same position during PAS. No other adverse effects were reported. Immediately after stimulation, we observed redness of the skin under the stimulating electrodes. This redness resulted from the increased blood flow in the stimulated region rather than from mechanical damage to the skin and disappeared in a few hours. No seizures were observed.

\section{DISCUSSION}

Spontaneous recovery 1.5 years after incomplete $\mathrm{SCl}$ is rare. ${ }^{20}$ In this proof-of-principle study, we demonstrate in two individuals with chronic incomplete $\mathrm{SCl}$ that long-term PAS is capable of returning some voluntary control over paralyzed muscles. Our study is the first applying PAS more than once to SCI patients. We are aware of only one report describing PAS delivered more than once to a group of neurological patients; in ambulatory chronic stroke patients, 4 weeks of PAS improved several gait measures. ${ }^{21}$ $\mathrm{SCl}$ patients may be more responsive to PAS than stroke patients; in stroke, the upper motor neurons are damaged, whereas in $\mathrm{SCl}$, both the upper and lower motor neurons can be preserved and only the connectivity between them needs to be re-established. Our results suggest that a stimulation period exceeding 4 weeks may be needed to observe the full effect.
We used trains of $50 \mathrm{~Hz}$ instead of commonly used single-pulse or $10-\mathrm{Hz}$ trains ${ }^{4}$ for PNS in PAS. The combination of TMS with single peripheral pulses leads to an LTP-like effect at a very limited range of ISIs; at ISIs outside this range, PAS leads to no effect or to long-term depression-like plasticity. ${ }^{4,10}$ In a long-term treatment, the initially calculated ISI would need to be constantly adjusted, as conductivity may change over time. Moreover, it is plausible that the residual fibers after $\mathrm{SCl}$ have a wide range of conductivities. A single high-intensity TMS pulse results in a high-frequency repetitive discharge of corticospinal neurons. ${ }^{22}$ The combination of high-intensity TMS pulses with $50-\mathrm{Hz}$ trains for the peripheral component of PAS could enable LTP-like effects at wider ranges of ISIs. Spike-time dependent plasticity is dependent not only on spike timing, but also on the firing rate, the number of coactive synaptic inputs and the postsynaptic voltage. ${ }^{23}$ The $20-\mathrm{ms}$ pulse interval in a stimulus train may increase the probability of some of the orthodromic and antidromic volleys arriving at the corticomotoneuronal synapses within the LTP-inducing window. When LTP-inducing and long-term depression-inducing timing interactions occur at the same time, LTP can override long-term depression. ${ }^{24}$ We demonstrate here in a healthy individual (see Methods scetion - control PNS) that PAS with $50 \mathrm{~Hz}$ peripheral component induces MEP facilitation at $1 \mathrm{~h}$ after stimulation, whereas control $50 \mathrm{~Hz}$ PNS does not; the absence of MEP facilitation immediately $(0 \mathrm{~min})$ after PAS most probably results from fatigue of the muscle induced by strong peripheral stimulation, corresponding to phenomenon observed in the patients.

We used high-intensity PNS to ensure its efficacy; in SCl patients the excitability of the peripheral neurons is low. ${ }^{25}$ Although our parameters are comparable to those used in routine clinical neurophysiology diagnostics and are considered safe, this stimulation intensity would be uncomfortable for a person with normal sensation. However, both patients tolerated it well, due to reduced or lack of sensation in the areas where stimulation was delivered.

Both patients were instructed to focus on the movements produced by the muscles innervated by the stimulated nerves during PAS and PNS. It has been previously shown that attention is critical for PAS-induced plasticity in human motor cortex. ${ }^{26}$ PAS procotol enhances the output of the corticospinal tract; it is possible that thinking about the corresponding movement during PAS protocol activates also secondary motor areas. Further research is required to dissect out whether mental concentration is required for reported therapeutic effects.

In patient $A$, the treatment was first given to left leg only; during this time, the right ankle remained paralyzed. In patient B, PNS and mental concentration on the movement of the left hand alone did not improve the motor score, whereas subsequent PAS of the same hand did improve motor performance. Importantly, we show that when PAS was later applied to the same hand, there was an improvement in the motor scores; this verifies that the combination of TMS, PNS and possibly mental concentration on the movement is responsible for the observed effect of the intervention. We did not include the control condition in which TMS would be used without PNS. Low-frequency TMS is, however, known to have an inhibitory effect, ${ }^{27}$ and thus it is highly improbable that TMS alone would have accounted for the obtained results.

In patient $B$, the muscles not innervated by the stimulated nerves showed robust improvement as well, whereas in patient $A$ there was no such effect. As the hand muscle function is highly interconnected, better performance of the muscles that were innervated by the stimulated nerves could have enabled the patient to use also the other muscles more effectively. It is also possible that high-intensity TMS and PNS enabled spread of the activation to adjacent cortical sites and peripheral nerves, respectively. 
Activation of some non-target muscles can be observed on the Supplementary Videos during motor performance. During clinical evaluations, the results of which are presented in this study, each muscle was assessed by the physiotherapist one-by-one with other muscles immobilized.

In both patients, the MEPs increased over time in all muscles tested, but no MEP increase was observed immediately after the PAS sessions (similar phenomenon was observed in the healthy control individual, see above). The most probable explanation is fatigue in the initially very weak muscles caused by the $50-\mathrm{Hz}$ high-intensity PNS.

The sensation scores were not improved. This is not surprising, because PAS specifically targeted the motor tracts. It remains to be seen whether long-term PAS protocols can be developed to also target the sensory tracts ${ }^{28}$ in $\mathrm{SCl}$ patients.

Our study was based on two patients with different levels of injury and has several limitations. It is a pilot proof-of-principle study and thus is open-labeled. The main outcome of the treatment was the appearance of the voluntary movement in the ankles of patient $A$ and the appearance of the ability to grasp objects in patient $\mathrm{B}$; the newly acquired voluntary movements did not lead to increase in SCIM scores (which reflect self-care capability). Nevertheless, even a weak movement, instead of no movement at all, will prevent problems associated with complete paralysis, such as the susceptibility to venous thrombosis, and may also have a psychological impact. Furthermore, our study leaves open the question of the optimal duration of the stimulation; it remains to be seen whether further neurological improvement could be achieved if the stimulation were continued for longer periods of time.

Both patients received conventional rehabilitation in parallel with the study; our research team did not influence the rehabilitation programs, and the exact role played by the rehabilitation in the observed effects remains open. The physical rehabilitation was not increased concurrently with the initiation of PAS. Patient A received no rehabilitation for the ankle muscles before the appearance of the movements; this effect was thus due to PAS only. Both patients received the same conventional rehabilitation for 2 years before the study, and there was no improvement in motor scores during the year before PAS.

In chronic complete $\mathrm{SCl}$, the surgical implantation of an epidural electrical stimulator enabled partial restoration of voluntary movement in paralyzed muscles when the stimulator was turned on. ${ }^{29,30}$ We have demonstrated that in incomplete chronic SCl, noninvasive stimulation can improve motor performance without surgery or robotic devices. The adult injured corticospinal tract has capacity for sprouting and adaptive reorganization at multiple levels. ${ }^{31,32}$ Our results justify further research of PAS applied at earlier stages after $\mathrm{SCl}$, before onset of irreversible muscle changes. ${ }^{33}$ Regeneration is only a first step in restoring function; the axons growing beyond the site of $\mathrm{SCl}$ need to make useful connections as well. ${ }^{1}$ Further investigation is warranted to explore whether PAS could enhance the benefit of other repair strategies. Modifying PAS parameters and possibly applying the stimulation for even longer periods of time may further improve the outcomes.

\section{ACKNOWLEDGEMENTS}

We are deeply grateful to our patients for their cooperation and patience. We thank David Montero-Danger, Suvi Lehto and Outi Nikkilä for excellent technical assistance and Andrey Zhdanov and Victor Pavlushkov for help with the MATLAB and Python. We thank Professor Eero Castrén for participation in evaluation of mechanism underlying the treatment. This study was supported by the Emil Aaltonen Foundation (AS), Maire Taponen Foundation (AS), Centre for International Mobility (AZ) and the SalWe Research Program for Mind and Body (Tekes-the Finnish Funding Agency for Technology and Innovation grant 1104/10; BioMag Laboratory).

\section{AUTHOR CONTRIBUTIONS}

Conception and design of the study by AS, PL, EK, SS, AY and JPM. Acquisition, analysis and interpretation of the data by AS, PL, AZ, NB, LK, EK, SS and JPM. Drafting of the manuscript by AS. All authors contributed to the critical revision of the manuscript for important intellectual content, approval of the final version and accountability for all aspects of the work.

\section{COMPETING INTERESTS}

PL reports personal fees from Nexstim Ltd., outside the submitted work. The remaining authors declare no conflict of interest.

\section{REFERENCES}

1 Ramer LM, Ramer MS, Bradbury EJ. Restoring function after spinal cord injury: towards clinical translation of experimental strategies. Lancet Neurol 2014; 1312: 1241-1256.

2 Dan Y, Poo MM. Spike timing-dependent plasticity of neural circuits. Neuron 2004; 441: 23-30.

3 Stefan K, Kunesch E, Cohen LG, Benecke R, Classen J. Induction of plasticity in the human motor cortex by paired associative stimulation. Brain 2000; 123: Pt 3572-Pt 3584.

4 Carson RG, Kennedy NC. Modulation of human corticospinal excitability by paired associative stimulation. Front Hum Neurosci 2013; 7: 823.

5 Taylor JL, Martin PG. Voluntary motor output is altered by spike-timingdependent changes in the human corticospinal pathway. J Neurosci 2009; 2937: $11708-11716$.

6 Cortes M, Thickbroom GW, Valls-Sole J, Pascual-Leone A, Edwards DJ. Spinal associative stimulation: a non-invasive stimulation paradigm to modulate spinal excitability. Clin Neurophysiol 2011; 12211: 2254-2259.

7 Leukel C, Taube W, Beck S, Schubert M. Pathway-specific plasticity in the human spinal cord. Eur J Neurosci 2012; 10: 1622-1629.

8 McKay DR, Ridding MC, Thompson PD, Miles TS. Induction of persistent changes in the organisation of the human motor cortex. Exp Brain Res 2002; 1433: 342-349.

9 Roy FD, Yang JF, Gorassini MA. Afferent regulation of leg motor cortex excitability after incomplete spinal cord injury. J Neurophysiol 2010; 1034: 2222-2233.

10 Bunday KL, Perez MA. Motor recovery after spinal cord injury enhanced by strengthening corticospinal synaptic transmission. Curr Biol 2012; 2224: 2355-2361.

11 Benito J, Kumru H, Murillo N, Costa U, Medina J, Tormos JM et al. Motor and gait improvement in patients with incomplete spinal cord injury induced by highfrequency repetitive transcranial magnetic stimulation. Top Spinal Cord Inj Rehabil 2012; 182: 106-112.

12 Belci M, Catley M, Husain M, Frankel HL, Davey NJ. Magnetic brain stimulation can improve clinical outcome in incomplete spinal cord injured patients. Spinal Cord 2004; 427: 417-419.

13 Ellaway PH, Vasquez N, Craggs M. Induction of central nervous system plasticity by repetitive transcranial magnetic stimulation to promote sensorimotor recovery in incomplete spinal cord injury. Front Integr Neurosci 2014; 8: 42.

14 Nardone R, Holler Y, Brigo F, Orioli A, Tezzon F, Schwenker K et al. Descending motor pathways and cortical physiology after spinal cord injury assessed by transcranial magnetic stimulation: a systematic review. Brain Res 2014; 1619: 139-154.

15 Field-Fote EC. Exciting recovery: augmenting practice with stimulation to optimize outcomes after spinal cord injury. Prog Brain Res 2015; 218: 103-126.

16 Beekhuizen KS, Field-Fote EC. Sensory stimulation augments the effects of massed practice training in persons with tetraplegia. Arch Phys Med Rehabil 2008; 894: 602-608.

17 Backus D, Cordo P, Gillott A, Kandilakis C, Mori M, Raslan AM. Assisted movement with proprioceptive stimulation reduces impairment and restores function in incomplete spinal cord injury. Arch Phys Med Rehabil 2014; 958: 1447-1453.

18 Gomes-Osman J, Field-Fote EC. Cortical vs afferent stimulation as an adjunct to functional task practice training: a randomized, comparative pilot study in people with cervical spinal cord injury. Clin Rehabil 2015; 298: 771-782.

19 Shulga A, Lioumis P, Kirveskari E, Savolainen S, Makela JP, Ylinen A. The use of F-response in defining interstimulus intervals appropriate for LTP-like plasticity induction in lower limb spinal paired associative stimulation. J Neurosci Methods 2015; 242: C112-C117.

20 Fawcett JW, Curt A, Steeves JD, Coleman WP, Tuszynski MH, Lammertse D et al. Guidelines for the conduct of clinical trials for spinal cord injury as developed by the ICCP panel: spontaneous recovery after spinal cord injury and 
statistical power needed for therapeutic clinical trials. Spinal Cord 2007; 453: 190-205.

21 Uy J, Ridding MC, Hillier S, Thompson PD, Miles TS. Does induction of plastic change in motor cortex improve leg function after stroke? Neurology 2003; 617: 982-984.

22 Di Lazzaro V, Ziemann U, Lemon RN. State of the art: Physiology of transcranial motor cortex stimulation. Brain Stimul 2008; 14: 345-362.

23 Feldman DE. The spike-timing dependence of plasticity. Neuron 2012; 754: 556-571.

24 Sjostrom PJ, Turrigiano GG, Nelson SB. Rate, timing, and cooperativity jointly determine cortical synaptic plasticity. Neuron 2001; 326: 1149-1164.

25 Dietz V. Behavior of spinal neurons deprived of supraspinal input. Nat Rev Neurol 2010; 63: 167-174.

26 Stefan K, Wycislo M, Classen J. Modulation of associative human motor cortical plasticity by attention. J Neurophysiol 2004; 921: 66-72.

27 Hallett M, Berrardelli A. Movement disorders. In: Epstein CM, Wassermann EM, Ziemann U (eds). Oxford Handbook of Transcranial Stimulation. Oxford University Press:New York, USA, 2008.
28 Wolters A, Schmidt A, Schramm A, Zeller D, Naumann M, Kunesch E et al. Timing-dependent plasticity in human primary somatosensory cortex. J Physiol 2005; 565: 1039-1052.

29 Harkema S, Gerasimenko Y, Hodes J, Burdick J, Angeli C, Chen Y et al. Effect of epidural stimulation of the lumbosacral spinal cord on voluntary movement, standing, and assisted stepping after motor complete paraplegia: a case study. Lancet 2011; 377: 1938-1947.

30 Angeli CA, Edgerton VR, Gerasimenko YP, Harkema SJ. Altering spinal cord excitability enables voluntary movements after chronic complete paralysis in humans. Brain 2014; 137: 1394-1409.

31 Raineteau O, Schwab ME. Plasticity of motor systems after incomplete spinal cord injury. Nat Rev Neurosci 2001; 4: 263-273.

32 Rosenzweig ES, Courtine G, Jindrich DL, Brock JH, Ferguson AR, Strand SC et al. Extensive spontaneous plasticity of corticospinal projections after primate spinal cord injury. Nat Neurosci 2010; 1312: 1505-1510.

33 Biering-Sorensen B, Kristensen IB, Kjaer M, Biering-Sorensen F. Muscle after spinal cord injury. Muscle Nerve 2009; 404: 499-519.

Supplemental Information accompanies the paper on the Spinal Cord Series and Cases website (http://www.nature.com/scsandc) 